\title{
Remote sensing of tropospheric turbulence using GPS radio occultation
}

\author{
Esayas Shume $^{1,2}$ and Chi Ao ${ }^{1}$ \\ ${ }^{1}$ Jet Propulsion Laboratory, California Institute of Technology, Pasadena, CA, USA \\ ${ }^{2}$ Department of Astronomy, California Institute of Technology, Pasadena, CA, USA \\ Correspondence to: Esayas Shume (esayas.b.shume@jpl.nasa.gov)
}

Received: 11 March 2016 - Published in Atmos. Meas. Tech. Discuss.: 20 April 2016

Revised: 16 June 2016 - Accepted: 19 June 2016 - Published: 21 July 2016

\begin{abstract}
Radio occultation (RO) measurements are sensitive to the small-scale irregularities in the atmosphere. In this study, we present a new technique to estimate tropospheric turbulence strength (namely, scintillation index) by analyzing RO amplitude fluctuations in impact parameter domain. GPS RO observations from the COSMIC (Constellation Observing System for Meteorology, Ionosphere, and Climate) satellites enabled us to calculate global maps of scintillation measures, revealing the seasonal, latitudinal, and longitudinal characteristics of the turbulent troposphere. Such information are both difficult and expensive to obtain especially over the oceans. To verify our approach, simulation experiments using the multiple phase screen (MPS) method were conducted. The results show that scintillation indices inferred from the MPS simulations are in good agreement with scintillation measures estimated from COSMIC observations.
\end{abstract}

\section{Copyright statement}

The JPL author's copyright for this publication is held by the California Institute of Technology. Government sponsorship acknowledged.

\section{Introduction}

Atmospheric turbulence associated with fluctuation of temperature, humidity, and water vapor are prevalent in the tropospheric region. Irregularities in the turbulence cause the index of refraction of the tropospheric medium to fluctuate. Electromagnetic signals transmitted from Global Navigation Satellite System (GNSS) satellites (for example), car- rying communication and navigation information, propagate through the turbulent troposphere. The spatial changes of the index of refraction introduce irregular fluctuations in the intensity and phase of the traversing electromagnetic signals by causing scintillation (Wheelon, 2004). Signal scintillation can affect the performance of satellite communication and navigation systems such as Global Positioning System (GPS). Scintillation characteristics inferred from global GPS signal observations (employed here) are valuable resources to study atmospheric turbulence properties.

In this paper, we have employed data analysis and model simulation to investigate and quantify the effects of tropospheric turbulence on L-band signals propagating from a GPS satellite to a low-Earth orbit (LEO) satellite such as COSMIC (Constellation Observing System for Meteorology, Ionosphere, and Climate) (Anthes et al., 2008). We estimated global distribution of the turbulence strength using a scintillation parameter (scintillation index) from COSMIC radio occultation (RO) measurements. To understand and quantify the observed scintillation, we have simulated the effect of tropospheric turbulence on L-band signals propagating through multiple phase screens (MPSs). In the MPS model runs, the phase screens are assumed to be various realizations of random perturbations of index of refraction profiles through which electromagnetic waves are propagating (Knepp, 1983).

\section{Global scintillation maps inferred from COSMIC RO measurements}

This section presents investigation of the effect of tropospheric turbulence on L-band propagation utilizing $\mathrm{RO}$ ob- 
servations from a GPS to a COSMIC satellites radio links. We estimated the impact of turbulence strength on L-band signals in terms of scintillation index. During the time frame relevant to this study, the COSMIC satellites provide a significant number of RO profiles (up to about 2000 profiles per day) observed by the six micro-satellites covering the entire globe. Utilizing the RO profiles, we were able to estimate the global distribution of the effect of scintillation on GPS signals. The technique provides valuable scintillation data especially over the oceans where ground-based measurements are both difficult and expensive to perform. RO data were first used to determine the intensity and location of turbulent regions (Cornman et al., 2012). Our study differs in that we aim to study the global climatology of tropospheric turbulence. In addition, we suggest that amplitude and phase in the impact parameter domain, rather than raw signal amplitude and phase, provide a more effective observable for measuring scintillation of interest.

\subsection{COSMIC signal amplitude measurements}

The basic observations of RO soundings on COSMIC are time series of amplitude (the $1 \mathrm{~s}$ voltage signal-to-noise ratio) and phase of L-band signals transmitted by a GPS satellite (e.g., Hajj et al., 2002). As the radio signal propagates through the troposphere, the amplitude of the signal (raw amplitude) suffers from the effects of defocusing, multi-path propagation, and diffraction effects. Therefore, the raw amplitude fluctuation does not truly represent the effect of turbulence. To suppress amplitude fluctuations due to these effects, the canonical transform (CT) has been applied on the received complex signal to transform it from the time domain to the impact parameter domain (Gorbunov, 2002). In RO retrieval processing, the CT phase is considered the important quantity since it is used to retrieve the bending angle profile and subsequently the refractivity profile, which are the primary retrieval variables. In our study, however, the focus is on the CT amplitude. We assume that CT amplitude fluctuations are dominated by small-scale irregularities caused by turbulent processes. We note that other radio-holographic inversion methods can be used such as the full spectrum inversion (Jensen et al., 2003) and phase matching method (Jensen et al., 2004). While these methods might be easier to implement for certain orbital geometry, studies have shown that all methods yield very similar results in phase and amplitude (Gorbunov et al., 2004).

We note that CT operates under the assumption of a spherically symmetric atmosphere where each ray is uniquely identified by its impact parameter. Thus the presence of mesoscale or large-scale horizontal inhomogeneity can result in fluctuations in the CT amplitudes; however, these tend to occur at a larger spatial scales than the turbulent effects being considered so that their contribution to the scintillation estimates is expected to be small.

\subsection{Scintillation effects on COSMIC RO signals}

The scintillation index $\sigma_{I}$ can be viewed as a measure of the effect of tropospheric turbulence on L-band propagation having signal intensity $I . \sigma_{I}$ can be described as a normalized standard deviation of intensity $I$ fluctuation:

$\sigma_{I}=\left(\frac{\left\langle I^{2}\right\rangle-\langle I\rangle^{2}}{\langle I\rangle^{2}}\right)^{\frac{1}{2}}$,

where $\langle\ldots\rangle$ stands for expected value and $I$ is the CT intensity, which is the square of the CT amplitude. The expected value is computed using data in $120 \mathrm{~m}$ intervals.

\subsubsection{Scintillation index estimates}

Figures 1 and 2 show global maps of $\sigma_{I}$ estimated by applying Eq. (1) on the CT intensity computed from the COSMIC RO measurements.

In the global map of scintillation estimates presented in Fig. 1, the scintillation index is an altitudinal average of $\sigma_{I}$ between altitudes 1 to $4 \mathrm{~km}$. Figure 2 shows an average of $\sigma_{I}$ between 4 and $8 \mathrm{~km}$. In this study, we restrict our results to above $1 \mathrm{~km}$ due to possible data quality issues affecting the near-surface retrievals (Ao et al., 2012). The global scintillation maps presented in Figs. 1 and 2 are for the months of January, April, July, and October of the year 2008. To construct these global scintillation maps, about 60000 RO profiles were employed. The results can be easily extended to other periods.

The scintillation maps (Figs. 1 and 2) contain a wealth of valuable information. Figures 1 and 2 show similar geographic and seasonal patterns; however, the values of $\sigma_{I}$ are significantly higher at the lower altitudes shown in Fig. 1. This is due to the sensitivity of the radio signals to the water vapor irregularities in the lower troposphere and as a result signal scintillations are more frequently observed between the top of the boundary layer and $4 \mathrm{~km}$ (cf. Fig. 3 of Sokolovskiy, 2001, and Fig. 2 of Beyerle et al., 2008, for example). From the scintillation maps, the followings can be noted.

1. The effects of the tropospheric turbulence on L-band propagation have a very strong seasonal dependence. Summer hemispheres show significant turbulent activities (measured by $\sigma_{I}$ ) compared to winter hemispheres (Figs. 1a, c and 2a, c). The magnitude of the global L-band scintillation is relatively large in the summer hemisphere compared to the winter hemisphere. Increased turbulence activities due to surface heating could be a reason for higher scintillation in the summer hemisphere. This property is also consistent with the global distribution of precipitation content, which has relatively significant magnitude during summer (Adler et al., 2003; Xie and Arkin, 1997). 

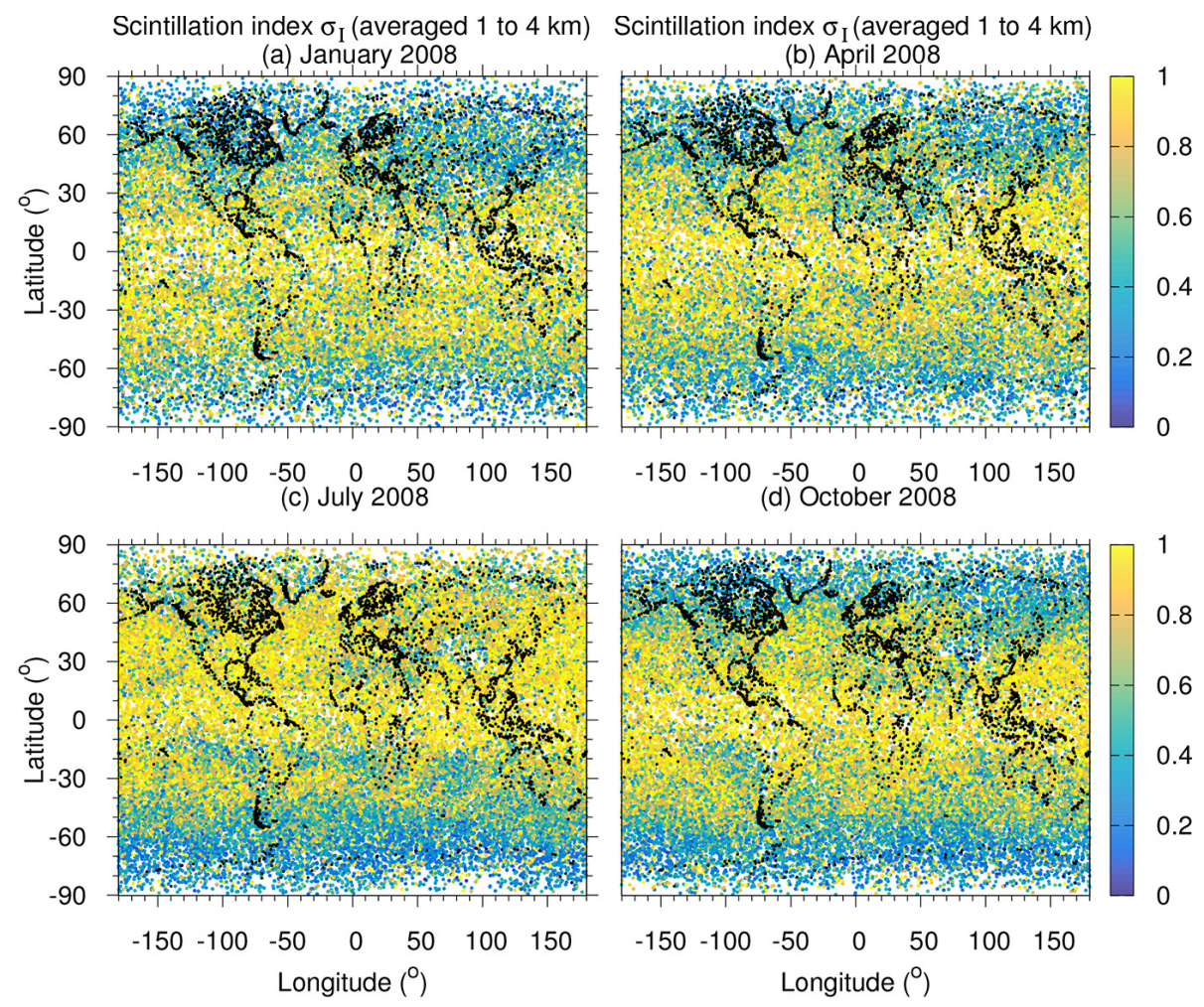

Figure 1. Global map of altitudinal average (1-4 km altitudes) scintillation index derived from the COSMIC RO data for January, April, July, and October 2008.

2. Figures 1 and 2 show that irrespective of seasons, the tropical regions are characterized by a relatively large $\sigma_{I}$ possibly due to the concentration of significant amount of water vapor in the lower tropical troposphere.

3. Using $\sigma_{I}$ as a proxy for turbulence strength, we infer that the northern hemispheric summer (July 2008, Figs. 1c and 2c) shows relatively large turbulent activities compared to the southern hemispheric summer (January 2008, Figs. 1a and 2a). During the respective summer months, the Antarctic region is characterized by less turbulence strength compared to the Arctic region. This is most pronounced in the temperate and polar regions. The effect of the Arctic sea and the high altitude of the Antarctic, make the Arctic polar region relatively warmer than the Antarctic region. The warm air in the Arctic holds more moisture than the cold air in the Antarctic. Temperature fluctuations could also cause the observed differences.

4. The scintillation estimates for the equinox seasons (April 2008, Figs. 1b and 2b; October 2008, Figs. 1d and $2 \mathrm{~d}$ ) are symmetrical about the equator. Scintillation estimates in the tropics are fairly symmetrical for all seasons.

5. The troposphere over the Sahara region is characterized by low scintillation effects on L-band propagation com- pared to the neighboring regions to its east and west. The water vapor content is consistently low and the air is dry over the Sahara (Schrijver et al., 2009). A close comparison of the maps (Figs. 1a-d and 2a-d) shows that the strength of scintillation is relatively enhanced during July (summer) over the Sahara due to relatively hotter air.

6. Figures $1 \mathrm{a}-\mathrm{d}$ and $2 \mathrm{a}-\mathrm{d}$ clearly demonstrate an oceancontinent contrast of the scintillation estimates in the Southeast Pacific, South America, and South Atlantic regions. Over these regions, the average $\sigma_{I}$ is low over the ocean compared to the continent. These $\sigma_{I}$ oscillations are highly correlated with (i) the convective available potential energy (CAPE), the values of which are maximum over the continent (Riemann-Campe et al., 2009), and (ii) atmospheric relative humidity from satellite data (Du et al., 2012).

7. The turbulence strength difference between summer and winter seasons over the Antarctica is small compared to the turbulence strength difference between summer and winter seasons over the Arctic. Similar results based on radiosonde, satellite, and atmospheric reanalyses observation were reported (Serreze et al., 2012; Bromwich et al., 2007). Compared to other regions, the Arctic and Antarctic have the least observations available. The RO 

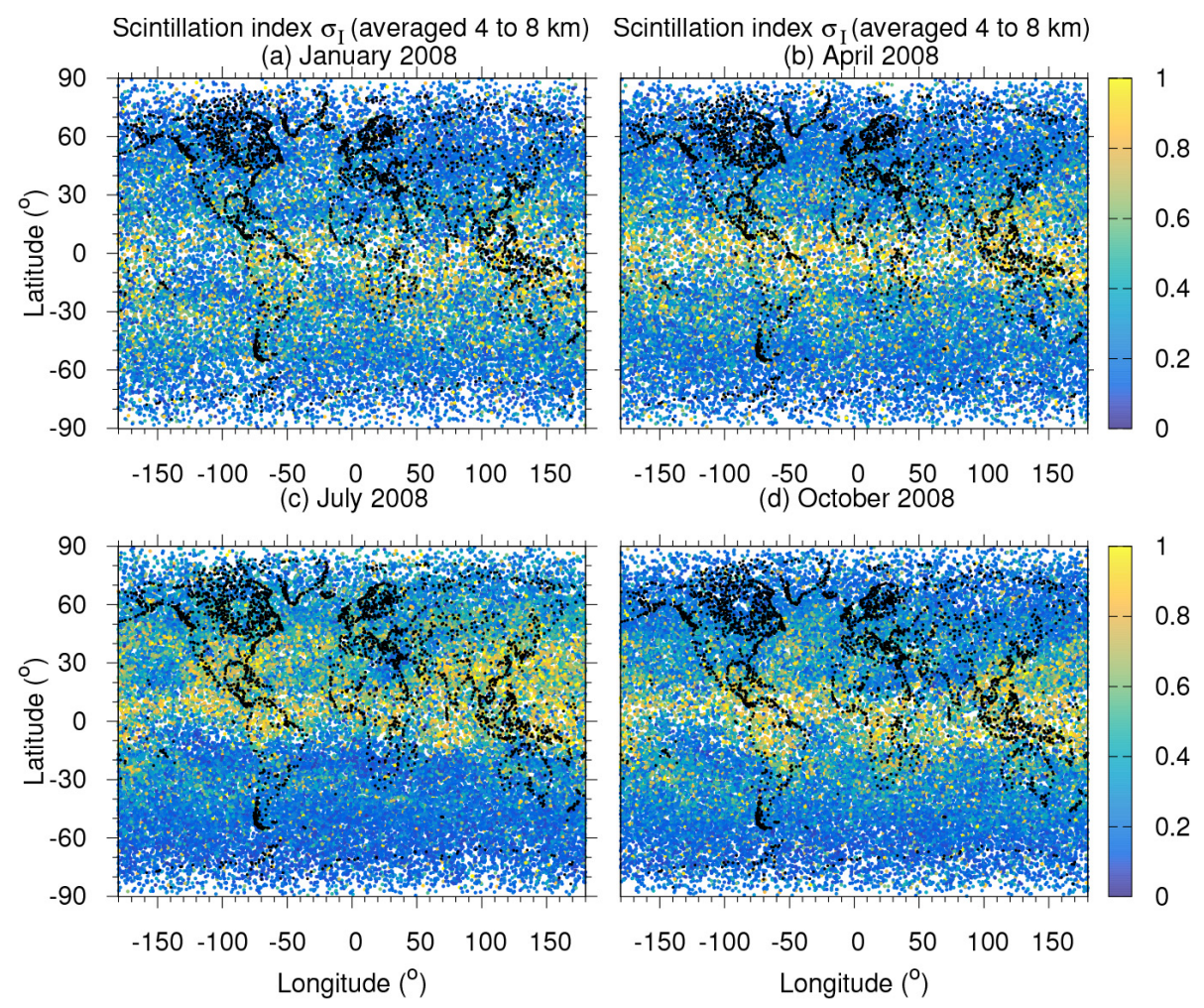

Figure 2. Global map of altitudinal average (4-8 km altitudes) scintillation index derived from the COSMIC RO data for January, April, July, and October 2008.
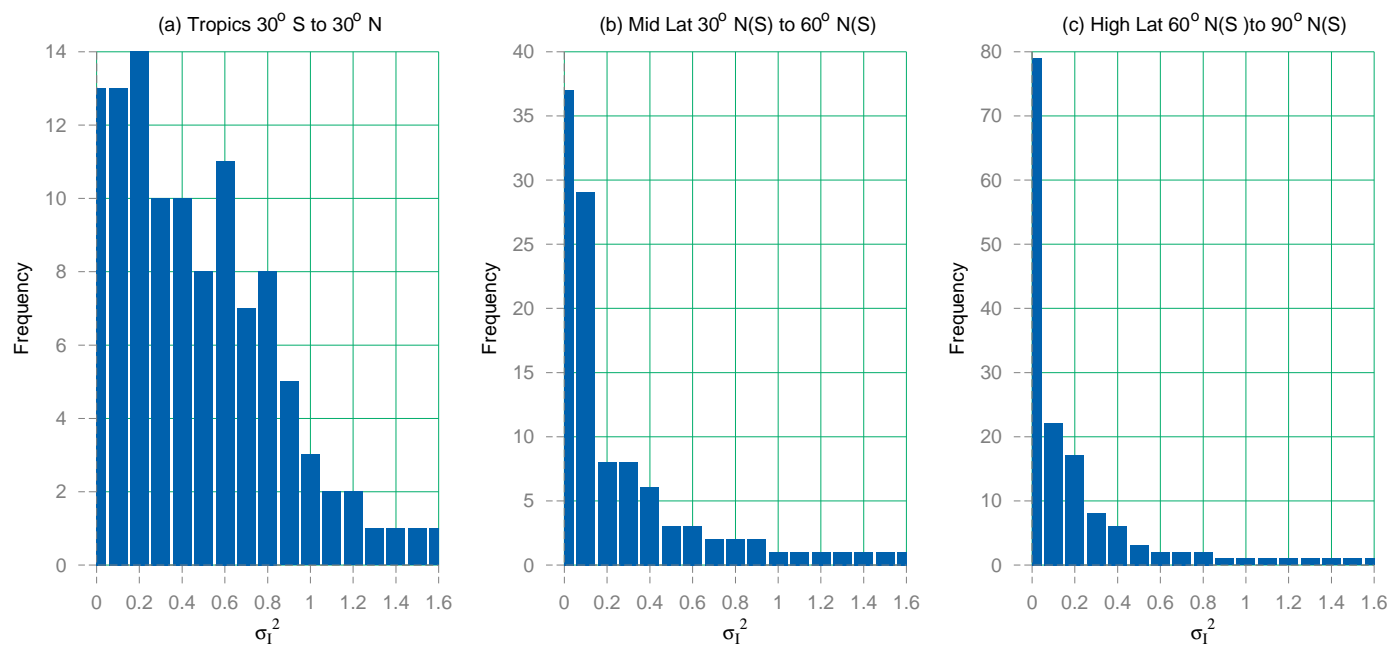

Figure 3. Histogram of $\sigma_{I}^{2}$ COSMIC RO averaged over 1-8 km: (a) tropics, (b) midlatitude, and (c) high latitude.

inferred map presented in Figs. 1 and 2, therefore, helps to build and enrich the polar region database.

8. For all seasons, $\sigma_{I}$ changes significantly with season in the Northeast Pacific compared to Southeast Pacific.

\subsubsection{The index of refraction structure parameter}

The index of refraction structure parameter $C_{n}^{2}$ is valuable for investigating propagation of electromagnetic waves in the atmosphere. Amplitude and phase of the waves propagating through the atmosphere get degraded as $C_{n}^{2}$ is intensified (Andreas, 1989). Unfortunately, measuring $C_{n}^{2}$ is expensive and difficult to perform, especially over the oceans. Due 
Index of refraction structure parameter $\mathrm{C}_{\mathrm{n}}^{2}$ (mean) COSMIC RO
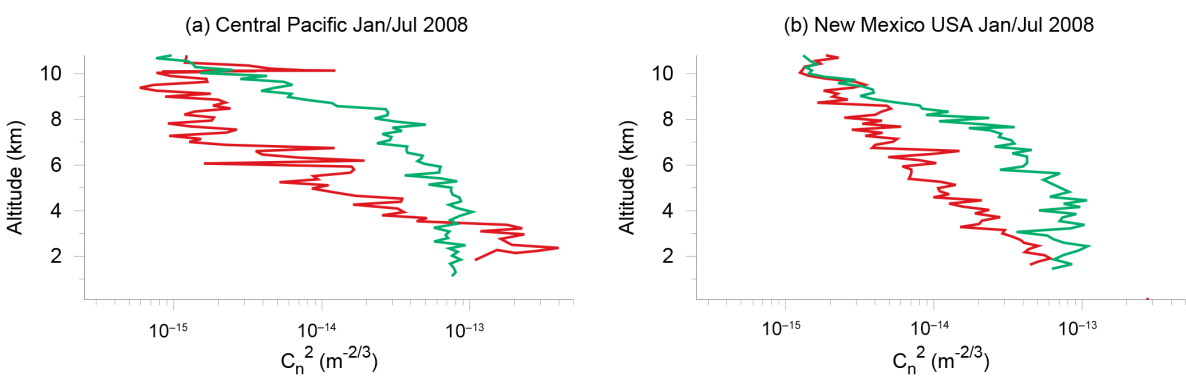

(c) South East Pacific Jan/Jul 2008
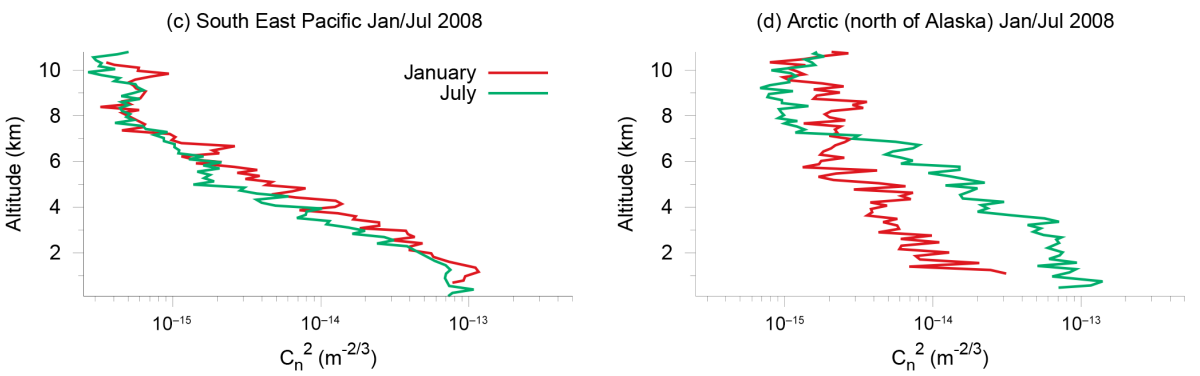

Figure 4. The index of refraction structure parameter $C_{n}^{2}$ inferred from CT signal amplitude of COSMIC RO data.

to this, $C_{n}^{2}$ profiles are available from only a few locations over the globe (Andreas, 1989; Wheelon, 2004 and the references). RO offers the possibility of filling these gaps. Here, we present $C_{n}^{2}$ profiles inverted from CT amplitude scintillation incurred on COSMIC RO observations and the Rytov variance (Eq. 2).

Assuming that the wavelength of propagation is small compared to the scale of index of refraction fluctuations, $\sigma_{I}$ can be expressed in terms of turbulence properties $\left(C_{n}^{2}\right)$ of the medium by the Rytov variance. The Rytov variance assumes wave propagation in weak fluctuation regimes $\left(\sigma_{I}^{2}<\right.$ 1) caused by turbulence processes having characteristics of Kolmogorov spectra. Since the amplitude of fluctuations of the RO signals considered are characterized by $\sigma_{I}^{2}<1$, the Rytov variance is valid for the present analysis. Note that the Rytov variance was used to describe characteristics of weak scintillation of radio and microwave propagation in the atmosphere (Clifford and Strohbehn, 1970; Woo and Ishimaru, 1973; Ishimaru, 1978; Martini et al., 2006; Blaunstein and Christodoulou, 2007). For plane waves, $\sigma_{I}^{2}$ is proportional to $C_{n}^{2}$ (Andrews et al., 1999):

$\sigma_{I}^{2}=1.23 C_{n}^{2} \kappa_{\circ}^{\frac{7}{6}} L^{\frac{11}{6}}$,

$C_{n}^{2}=0.813 \sigma_{I}^{2} \kappa_{\circ}^{-\frac{7}{6}} L^{-\frac{11}{6}}, \quad \kappa_{\circ}=\frac{2 \pi}{\lambda}$.

$\kappa_{\circ}$ is a wavenumber of the electromagnetic wave, $\lambda$ is wavelength, and $L$ is the propagation path length between a transmitter and a receiver through the turbulent medium.

The $C_{n}^{2}$ profile can be calculated from $\sigma_{I}^{2}$ profile for each occultation by making use of Eq. (2) with $\lambda=0.2 \mathrm{~m}$ and $\kappa_{o}=$
$31.4 \mathrm{~m}^{-1}$. Figure $3 \mathrm{a}, \mathrm{b}$, and $\mathrm{c}$ show the distribution of $\sigma_{I}^{2}$ averaged over $1-8 \mathrm{~km}$ in the tropics, midlatitude, and highlatitude troposphere, respectively. The histograms reveal that about $95 \%$ of the $\sigma_{I}^{2}$ values are less than 1 in Fig. 3a. The distribution of $\sigma_{I}^{2}$ shows values less than 1 for more than $95 \%$ of the cases. Since measured $\sigma_{I}^{2}<1$ in Figs. 1 and 2 predominantly, the application of the Rytov variance is justified for estimating $C_{n}^{2}$ profile. To compute $C_{n}^{2}$ profiles from $\sigma_{I}^{2}$ profiles, we still have to know the value of an effective signal propagation length $L$ in the lower troposphere corresponding to RO geometry. For that purpose, $L$ has been estimated using an MPS model simulation technique described in Sect. 3. We use the MPS model to calculate the scintillation index $\sigma_{I}^{2 \text { model }}$ by varying the value of $C_{n}^{2}$ (the MPS model runs use index of refraction as input derived from Kolmogorov spectra, Eqs. 3 and 4). We used the following procedures in the MPS model to estimate $L$. The relationship between the scintillation index $\sigma_{I}^{2 \text { model }}$ and $C_{n}^{2}$ is linear (Eq. 2). Using linear regression, we determine the slope in the $\sigma_{I}^{2}{ }^{\text {model }}$ and $C_{n}^{2}$ relationship. The propagation path length $L$ is estimated from the slope and has an average value of $L=650 \mathrm{~km}$ in the lower troposphere. We recognize that $L$ is not exactly a constant and would vary with altitudes and differ from sounding to sounding; however, we expect using a single average $L$ to estimate $C_{n}^{2}$ for all occultations to be a good approximation to first order.

Figure 4 depicts representative $C_{n}^{2}$ profiles calculated utilizing COSMIC RO signal amplitudes received over the Central Pacific, New Mexico, Southeast Pacific, and Arctic (north of Alaska) in January and July 2008. The $C_{n}^{2}$ profiles 

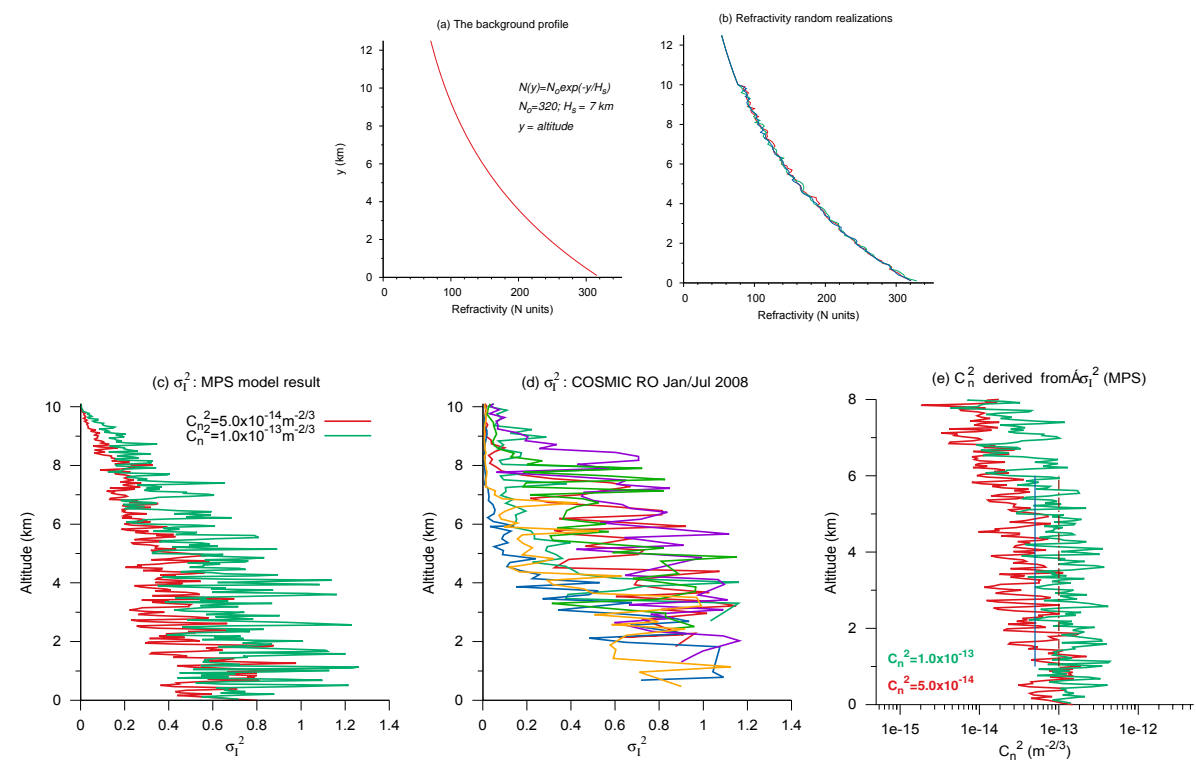

Figure 5. (a, b) Example refractivity profile employed as an input for the multi phase screen (MPS) simulation runs; (c) scintillation index $\sigma_{I}^{2}$ profiles inferred from MPS model runs; (d) $\sigma_{I}^{2}$ inferred from COSMIC RO observations (averages over Central Pacific, New Mexico, and Arctic, north of Alaska); and (e) $C_{n}^{2}$ derived from $\sigma_{I}^{2}$ MPS model.

are averaged over $5^{\circ}$ latitude and $5^{\circ}$ longitude. The $C_{n}^{2}$ profiles shown in Fig. 4, estimated from COSMIC RO, decrease with altitude in the lower troposphere in agreement with measured $C_{n}^{2}$ profiles (Andreas, 1989; Wheelon, 2004, for example). Specifically, in Fig. 4a, b, and d (Central Pacific, New Mexico, and Arctic (north of Alaska)), the $C_{n}^{2}$ profiles show clear seasonal behavior (summer-winter contrast). $C_{n}^{2}$ is larger in the July compared to January (New Mexico and Arctic (north of Alaska), Fig. 4b and d). It is larger in January compared to July (Southeast Pacific, Fig. 4c). In contrast, $C_{n}^{2}$ is only a little larger in July compared to January (Central Pacific, Fig. $4 \mathrm{a})$. The mean $C_{n}^{2}$ value $\left(0.25 \times 10^{-14} \mathrm{~m}^{-2 / 3}\right)$, reported in (Wheelon, 2004, and the references therein), agrees with an approximate mean summer values of $C_{n}^{2}$ displayed in Fig. $4 \mathrm{~b}$ and d.

\section{Wave propagation simulations}

\subsection{MPS model}

The merit of the phase screen technique to find solution of electromagnetic wave propagation through turbulent medium has been described in the literature (e.g., Knepp, 1983; Knepp and Nickisch, 2009). In the context of this paper, the MPS model is applied to simulate and quantify the effect of tropospheric turbulence (and the resulting index of refraction variations) on L-band propagation. The MPS technique employed uses the geometry of L-band propagation in a radio link from a GPS satellite (incident wave) to a LEO satellite (receiver, observation screen) (Sokolovskiy, 2001).
The MPS technique involves finding the solution of the parabolic wave equation (Knepp, 1983; Knepp and Nickisch, 2009). The solution comprises application of numerical and Fourier transform techniques repeatedly for waves propagating through MPSs (Knepp, 1983; Knepp and Nickisch, 2009) idealized by parallel planes (phase changing screens). The medium of signal propagation is divided into a number of thin layers, each modeled as phase changing screens encompassed by a free space. Accordingly, we seek solution of the parabolic equation by performing successive iteration of the propagation calculations from one screen to the next and ultimately to the observation screen.

\subsection{Model results and discussion}

\subsubsection{Index of refraction profiles: model input}

The main input for the MPS model run is an index of refraction profile of the lower troposphere. The phase screens are constructed as random perturbations of an exponential background refractivity profile (first term in Eq. 3):

$N(y)=N_{\circ} \exp \left(-\frac{y}{H_{\mathrm{s}}}\right)+\widetilde{N}(y)$,

where the index of refraction profile $n(y)$ is calculated from the refractivity profile using $N(y)=10^{6}[n(y)-1], N(0)=$ $N_{\circ}=320$, and the scale height $H_{\mathrm{s}}=7 \mathrm{~km}$. The resulting background refractivity is shown in Fig. 5a. In the refractivity perturbation models $(\widetilde{N}(y))$, tropospheric turbulence is taken to be nonzero only up to $8 \mathrm{~km}$. $\widetilde{N}(y)$ is an inverse Fourier transform of the Kolmogorov spectra (Eq. 4) modulated by 
a Gaussian random number with zero mean and standard deviation one (Knepp, 1983). In order to make the refractivity perturbations realistic, the refractivity profiles on the phase changing screens are specified by the characteristics of Kolmogorov spectra:

$$
\hat{\Phi}(\kappa)=0.033 C_{n}^{2} \kappa^{-\frac{11}{3}}, \quad \kappa=\frac{2 \pi}{y} .
$$

Figure $5 b$ shows example refractivity profiles calculated using Eq. (4) (an input for the MPS model runs).

\subsubsection{Input parameters for MPS model runs}

The input parameters for MPS model runs were as follows: the number of phase screens was equal to 4000 ; vertical spacing was $1 \mathrm{~m}$; spacing between phase screens was $1 \mathrm{~km}$; a Gaussian random number initializes each phase screen differently; on each phase screen, each altitude is initialized differently.

\subsubsection{Scintillation index profiles: MPS model}

The MPS model runs were performed for two cases where (a) $C_{n}^{2}=1.0 \times 10^{-13} \mathrm{~m}^{-2 / 3}$ and (b) $C_{n}^{2}=5.0 \times 10^{-14} \mathrm{~m}^{-2 / 3}$. For each case, 50 random realizations of the MPS runs were used to calculate CT amplitude profiles. The average CT intensity profiles (average of the 50 CT intensity profiles) were then used to calculate $\sigma_{I}$ profiles every $50 \mathrm{~m}$ in the lower troposphere (shown in Fig. 5c).

For comparison and validation purposes, Fig. 5d plots $\sigma_{I}$ profiles estimated from COSMIC RO data over Central Pacific, New Mexico, Arctic (north of Alaska), and Southeast Pacific. The MPS inferred $\sigma_{I}$ profiles are in a reasonable agreement with the COSMIC RO inferred $\sigma_{I}$. Figure $5 \mathrm{c}$ and d show a very good agreement between the MPS model $\sigma_{I}$ and COSMIC RO $\sigma_{I}$ over the Central Pacific (January and July 2008) and New Mexico (July 2008). Figure 5e presents $C_{n}^{2}$ estimates derived from $\sigma_{I}^{2}$ profiles (MPS model, Fig. 5c). The Rytov variance has been used to invert for the $C_{n}^{2}$ profiles. Figure $5 \mathrm{e}$ reproduces the input $C_{n}^{2}$ values fairly well. The $C_{n}^{2}$ profiles shown in Fig. 5e have similarities with the $C_{n}^{2}$ profiles shown in Fig. 4 validating $C_{n}^{2}$ profiles derived from COSMIC RO data. In Fig. 5e, the blue vertical line shows $C_{n}^{2}=5.0 \times 10^{-14} \mathrm{~m}^{-2 / 3}$ and the brown vertical line shows $C_{n}^{2}=1.0 \times 10^{-14} \mathrm{~m}^{-2 / 3}$. The decrease of $C_{n}^{2}$ starting $\sim 6 \mathrm{~km}$ is believed to be due to the finite vertical extent of the turbulence model in the MPS simulation.

\section{Conclusions}

We have used (i) radio occultation observations on board the COSMIC satellites and (ii) multiple phase screen model calculations to investigate and quantify the effect of tropospheric turbulence on L-band propagation. Instead of regular amplitude and phase data, we have used the CT amplitudes in estimating the scintillation indices from each occultation. This has the advantage of removing signal fluctuations due to atmospheric multi-path and diffraction from sharp vertical layers.

Global maps of scintillation measures across different seasons were obtained from 1 year of COSMIC RO data. The resulting global scintillation maps reveal very strong seasonal dependence, with the northern hemispheric summer exhibiting relatively large turbulent activities compared to the southern hemispheric summer. Irrespective of seasons, the tropical regions are generally characterized by a relatively large scintillation index. The maps also show clear ocean-continent contrast of the scintillation estimates in the Southeast Pacific, South America, and South Atlantic regions. The scintillation estimates appear to be positively correlated with water vapor, precipitation, and convection. We have also presented $C_{n}^{2}$ profiles estimated from COSMIC RO data using the Rytov variance method for weak scintillation. This represents the first ever observational estimates of global tropospheric turbulence strength. While certain simplifications have been used in this initial study, the results are encouraging, and future work can be done to refine the results and cross-validate with other observations.

We have also performed numerical simulations of radio propagation through a random phase changing screen (in which refractivity profiles were specified by the Kolmogorov spectra). Scintillation index profiles inferred from the MPS technique are in a reasonable agreement with scintillation index profiles inferred from COSMIC RO data and provide confidence to our estimates of $C_{n}^{2}$ profiles.

\section{Data availability}

The COSMIC radio occultation data used in this study were processed at the Jet Propulsion Laboratory (JPL) and are available from http://genesis.jpl.nasa.gov.

Author contributions. Esayas Shume and Chi Ao contributed to COSMIC RO data analysis, multiple phase screen simulation, and writing this paper.

Acknowledgements. The research described in this paper was carried out at the Jet Propulsion Laboratory, California Institute of Technology, under a contract with the National Aeronautics and Space Administration. The authors would like to acknowledge grant support from NASA ROSES GNSS Remote Sensing Team (NNH11ZDA001N-GNSS). We thank the UCAR COSMIC Data analysis and Archive Center for access to the COSMIC raw data.

Edited by: S. Malinowsk 


\section{References}

Adler, R. F., Huffman, G. J., Chang, A., Ferraro, R., Xie, P.-P., Janowiak, J., Rudolf, B., Schneider, U., Curtis, S., Bolvin, D., Gruber, A., Susskind, J., Arkin, P., and Nelkin, E.: The Version-2 Global Precipitation Climatology Project (GPCP) Monthly Precipitation Analysis (1979-Present), J. Hydrometeor, 4, 11471167, 2003.

Andreas, E. L.: The refractive index structure parameter, $C_{n}^{2}$, for a year over the frozen Beaufort Sea, Radio Sci., 24, 667-679, doi:10.1029/RS024i005p00667, 1989.

Andrews, L. C., Phillips, R. L., Hopen, C. Y., and Al-Hahash, M. A.: Theory of optical scintillation, J. Opt. Soc. Am. A, 16, 14171429, 1999.

Anthes, R. A., Ector, D., Hunt, D. C., Kuo, Y.-H., Rocken, C., Schreiner, W. S., Sokolovskiy, S. V., Syndergaard, S., Wee, T.K., Zeng, Z., Bernhardt, P. A., Dymond, K. F., Chen, Y., Liu, H., Manning, K., Randel, W. J., Trenberth, K. E., Cucurull, L., Healy, S. B., Ho, S.-P., McCormick, C., Meehan, T. K., Thompson, D. C., and Yen, N. L.: The COSMIC/FORMOSAT-3 Mission: Early Results, B. Am. Meteor. Soc., 89, 313-333, doi:10.1175/BAMS89-3-313, 2008.

Ao, C. O., Waliser, D. E., Chan, S. K., Li, J.-L., Tian, B., Xie, F., and Mannucci, A. J.: Planetary boundary layer heights from GPS radio occultation refractivity and humidity profiles, J. Geophys. Res., 117, D16117, doi:10.1029/2012JD017598, 2012.

Beyerle, G., Gorbunov, M. E., and Ao, C. O.: Simulation studies of GPS radio occultation measurements, Radio Sci., 38, 1084, doi:10.1029/2002RS002800, 2003.

Blaunstein, N. and Christodoulou C.: Radio Propagation and Adaptive Antennas for Wireless Communication Links Terrestrial, Atmospheric and Ionospheric Wiley Interscience, John Wiley And Sons, Inc., 2007.

Bromwich, D. H., Fogt, R. L., Hodges, K. I., and Walsh J. E.: A tropospheric assessment of the ERA-40, NCEP, and JRA-25 global reanalyses in the polar regions, J. Geophys. Res., 112, D10111, doi:10.1029/2006JD007859, 2007.

Clifford, S. F. and Strohbehn, J. W.: The theory of microwave line-of-sight propagation through a turbulent atmosphere, IEEE Trans. Antenn. Prop., AP-18, 264-274, 1970.

Cornman, L. B., Goodrich, R. K., Axelrad, P., and Barlow, E.: Progress in turbulence detection via GNSS occultation data, Atmos. Meas. Tech., 5, 789-808, doi:10.5194/amt-5-789-2012, 2012.

Du, J., Cooper, F., and Fueglistaler, S.: Statistical analysis of global variations of atmospheric relative humidity as observed by AIRS, J. Geophys. Res.-Atmos., 117, D12315, doi:10.1029/2012JD017550, 2012.

Gifford, F. A.: A Similarity Theory of the Tropospheric Turbulence Energy Spectrum, J. Atmos. Sci., 45, 1370-1379. doi:10.1175/1520-0469(1988)045<1370:ASTOTT>2.0.CO;2, 1988.

Gorbunov, M. E.: Canonical transform method for processing radio occultation data in the lower troposphere, Radio Sci., 37, 1076, doi:10.1029/2000RS002592, 2002.

Gorbunov, M. E., Benzon, H.-H., Jensen, A. S., Lohmann, M. S., and Nielsen, A. S.: Comparative analysis of radio occultation processing approaches based on Fourier integral operators, Radio Sci., 39, RS6004, doi:10.1029/2003RS002916, 2004.
Hajj, G. A., Kursinski, E. R., Romans, L. J., Bertiger, W. I., and Leroy S. S.: A technical description of atmospheric sounding by GPS occultation, J. Atmos. Sol.-Terr. Phy., 64, 451-469, doi:10.1016/S1364-6826(01)00114-6, 2002.

Ishimaru, A.: Wave Propagation and Scattering in Random Media, Multiple Scattering, Turbulence, Rough Surfaces, and Remote Sensing, Academic Press, New York San Francisco London, Vol. 2, 1978

Jensen, A. S., Lohmann, M. S., Benzon, H., and Nielsen, A. S.: Full spectrum inversion of radio occultation signals, Radio Sci., 38, 1040, doi:10.1029/2002RS002763, 2003.

Jensen, A. S., Lohmann, M. S., Nielsen, A. S., and Benzon, H.: Geometric optics phase matching of radio occultation signals, Radio Sci., 39, RS3009, doi:10.1029/2003RS002899, 2004.

Knepp, D. L.: Multiple phase-screen calculation of the temporal behavior of stochastic waves, P. IEEE, 71, 722-737, doi:10.1109/PROC.1983.12660, 1983.

Knepp, D. L. and Nickisch L. J.: Multiple phase screen calculation of wide bandwidth propagation, Radio Sci., 44, RS0A09, doi:10.1029/2008RS004054, 2009.

Martini, E., Freni, A., and Cuccoli, F.: Impact of Tropospheric Scintillation in the $\mathrm{Ku} / \mathrm{K}$ Bands on the Communications Between Two LEO Satellites in a Radio Occultation Geometry, IEEE T. Geosci. Remote, 44, 2062-2071, 2006.

Riemann-Campe, K., Fraedrich, K., and Lunkeit, F.: Global climatology of convective available potential energy (CAPE) and convective inhibition (CIN) in ERA-40 reanalysis, Atmos. Res., 93, 534-545, doi:10.1016/j.atmosres.2008.09.037, 2009.

Schrijver, H., Gloudemans, A. M. S., Frankenberg, C., and Aben, I.: Water vapour total columns from SCIAMACHY spectra in the $2.36 \mu \mathrm{m}$ window, Atmos. Meas. Tech., 2, 561-571, doi:10.5194/amt-2-561-2009, 2009.

Serreze, M. C., Barrett, A. P., and Stroeve, J.:, Recent changes in tropospheric water vapor over the Arctic as assessed from radiosondes and atmospheric reanalyses, J. Geophys. Res., 117, D10104, doi:10.1029/2011JD017421, 2012.

Sokolovskiy, S. V.: Modeling and inverting radio occultation signals in the moist troposphere, Radio Sci., 36, 441-458, doi:10.1029/1999RS002273, 2001.

Sokolovskiy, S. V., Rocken, C., Lenschow, D. H., Kuo, Y.-H., Anthes, R. A., Schreiner, W. S., and Hunt, D. C.: Observing the moist troposphere with radio occultation signals from COSMIC, Geophys. Res. Lett., 34, L18802, doi:10.1029/2007GL030458, 2007.

Wheelon, A.: Electromagnetic Scintillation - I. Geometric Optics, Cambridge University Press, Cambridge, UK, 2004.

Woo, R. and Ishimaru, A.: Remote sensing of the turbulence characteristics of a planetary atmosphere by radio occultation of a space probe, Radio Sci., 8, 103-108, doi:10.1029/RS008i002p00103, 1973.

Xie, P. and Arkin, P. A.: Global Precipitation: A 17-Year Monthly Analysis Based on Gauge Observations, Satellite Estimates, and Numerical Model Outputs, B. Am. Meteor. Soc., 78, 2539-2558, 1997. 